\title{
Primary and secondary punishment of toe sucking in the infant rhesus monkey'
}

H. Kaye, J. Cox, T. Bosack and K. Anderson BROWN UNIVERSITY

\begin{abstract}
A 10-week-old rhesus monkey was given a mild electric shock contingent on sucking her thumb or large toe. This totally suppressed sucking during several hours of testing, and apparently reduced sucking in the home cage immediately after testing. A sound which was inadvertently paired with shock during training was used during extinction of sucking-suppression to reduce the occurrence of another behavior. This indicated that sound had become a secondary punishing stimulus. Extinction procedures brought a return of sucking in both test and home environments.
\end{abstract}

\section{Problem}

Two recent experiments dealing with operantpunishment of toe- and thumb-sucking, through removal of positive reinforcement in one case, and presentation of noxious stimuli in another, have yielded partial or total suppression of this behavior. Baer (1962) withdrew a movie cartoon contingent on thumb-sucking in a 5-year-old child. Tolman and Mueller (1964) investigated suppression of sucking in a 16-month-old chronic toe-sucking rhesus monkey and replicated the Baer effect by showing partial suppression. In a second phase of the experiment, they used traditional noxious stimulus-response contingencies, with a "loud" complex noise, and showed complete suppression. Neither study indicates whether generalization occurred to environments other than the experimental situation.

The present study obtained operant aversive conditioning in a rhesus infant with a toe-sucking habit, and tested the generalization of this suppression to the home cage.

\section{Method}

The subject was a female rhesus monkey (Macaca mulatta) 75 days old at the beginning of the experiment. She had been separated from her mother at birth and was housed in a plexiglass cage separate from the rest of the rhesus colony. The test cage, in an adjacent room, was 1 cubic foot in size. It had a grid floor, below which was a pan containing sawdust. One side of the chamber was transparent to allow observation, the remainder opaque. The infant was tested one hour per day in the experimental chamber for 20 consecutive days: 7 days of baseline observation, 7 of conditioning, and 6 of extinction. Observations were also made of home-cage sucking for $30 \mathrm{~min}$. following the 13 conditioning and extinction days, and for 12 separate $15 \mathrm{~min}$. intervals, at random times, on baseline days.

During baseline, cumulative recordings were made of the number of 3 sec. intervals of sucking, as done by Baer and by Tolman and Mueller. An industrial timer with a 3-sec. timing cam provided the signal for manually stepping the cumulative recorder. The timer also was used to trip a counter to measure the total amount of time spent sucking.

The conditioning procedure consisted of alternating 15-min. punishment periods in which shock was contingent on sucking and 15-min. test periods of no shock. Two test and two punishment periods were given during each session. Brief shocks of 0.75

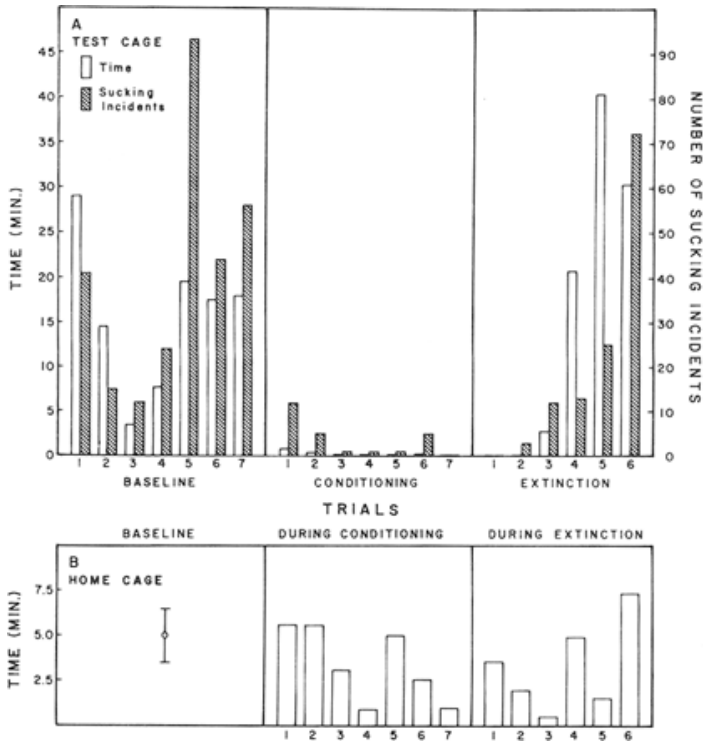

Fig. 1A. Amount of accumulated sucking time (open bars) and number of separate sucking incidents (striped bars) throughout the three phases of testing. Fig. 1B. Amount of time spent in toesucking during the first $15 \mathrm{~min}$. in the home cage after a test session. The baseline point represents the mean of twelve $15 \mathrm{~min}$. sessions, $\pm 1 \mathrm{SD}$.

ma were delivered manually from an Applegate Constant Current Stimulator (Model 250).

\section{Results and Diseussion}

Figure 1A shows the changes in number of separate sucking incidents and total time spent sucking during each day of testing. Sucking decreased sharply on the first conditioning day, and this suppression was maintained throughout conditioning and in early extinction. Eight punishments were delivered on Day 1 of conditioning, 6 in the first punishment period. Five more sucking-contingent shocks were given throughout the remainder of conditioning. Twelve other sucking incidents occurred at scattered times during the test periods of the 7 conditioning sessions, these lasting only fractions of a second.

Figure 1B shows that home-cage sucking decreased during conditioning and returned during extinction. These changes, however, were more variable, and aside from Days 4 and 7 of conditioning, and 2 and 3 of extinction, were much less dramatic than the testcage suppression and recovery.

Almost all sucking in the test cage was done on the large toe during baseline and conditioning, except for Day 6 of conditioning, in which all attempted sucks were on the thumb. During extinction the 3 sucking incidents in Day 2 were on the thumb, Day 3 involved 
both toe and thumb, but only the toe was sucked thereafter.

Mouthing activities (i. e., chewing the grid floor and sides of the cage), and abortive toe sucking (i. e., lifting toe toward mouth and stopping just short of lips), increased markedly from baseline to conditioning.

Because of space limitation, the timer was housed in the room with the experimental cage, producing a low-intensity, rasping sound each time it was activated. It was turned on each time the infant sucked its toe, and was always turned on, therefore, just before shock. As conditioning progressed, turning on the timer during test periods was observed to elicit reactions similar to those elicited by shock, i. e., leaping and immediate withdrawal of the thumb or toe from the mouth, followed by cringing, crying and grimacing. It was hypothesized that the timer noise had become a secondary punishing stimulus through classical conditioning. To test this hypothesis, timer noise was made contingent on another response, reaching through the grid floor to the sawdust pan below. The monkey had come to occupy more and more time with this activity, which was designated "pan play." During the last two conditioning sessions, cumulative recordings were taken of reaching through the grid floor and touching the sawdust pan or contents, each swipe of the hand being considered one response. The records from these two days served as baseline performance, to which secondary conditioning could be compared. During secondary punishment stop watches were used to record sucking time, with incidents being marked on the cumulative recorder via the event marker.

Figure 2 shows the frequency of "pan play" for the two days of baseline and six days of secondary punishment, the latter being the days in which suppression of toe- and thumb-sucking was being extinguished. Response frequency decreased greatly during the first day of punishment, but later increased during both punishment and test periods. Responding returned more slowly during punishment periods than in test periods. These results confirmed the hypothesis that the timer sound had secondary punishing properties. In addition, the phenomenon of secondary punishment helps account for the rapid and sustained suppression of sucking during conditioning since it multiplies the number of aversive stimulations given and broadens the "punishment" presentation time to both conditioning and test segments of any conditioning session. Pairing the timer

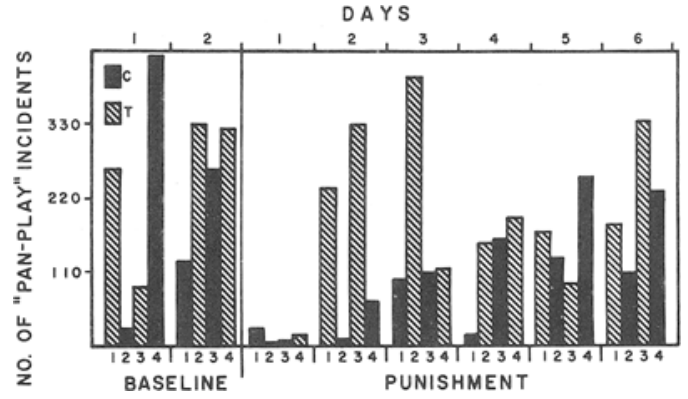

Fig. 2. "Pan play" in consecutive 15 min. segments on separate experimental days. During baseline, $\mathrm{T}$ represents "pan play"during non-shock periods, and $C$ represents periods in which shock was contingent on toe sucking. During punishment, $\mathrm{T}$ and $\mathrm{C}$ refer to nonbuzzer and buzzer periods, respectively.

sound with sucking during baseline may have acted as pretraining, facilitating the association of shock and timer noise during the conditioning sessions. In any event, the use of the timer in baseline indicates that the timer noise was not at the outset a punishing stimulus.

Presenting the timer sound not contingent on sucking during extinction may have produced a more rapid recovery of this suppressed response than would have been obtained by simply eliminating shock, since there were many more separate incidents of turning on the timer when using it to punish "pan play" than would have occurred had sucking continued to be recorded, and thus punished, with it.

Finally, although it is not possible to compare the differential effects of intensity of punishment, certain differences between our study and that of Tolman and Mueller might be worth pursuing. The trend of generalization to home-cage environment, and the rapidity of the occurrence of primary and secondary punishment effects may indicate that younger rhesus infants learn and retain punishment conditioning of a gross motor response better than the older rhesus.

\section{Referenees}

BAER, D. M. Laboratory control of thumbsucking by withdrawal and representation of reinforcement. J. exp. Anal. Behav., 1962, 5, 525-528.

TOLMAN, C. W., \& MUELLER, M. R. Laboratory control of toesucking in a young rhesus monkey by two kinds of punishment. J. exp. Anal. Behav., 1964, 7, 323-325.

Note

1. This research was supported in part by Public Health Service Research Grants MH07136 and NB04268 to Dr. A. M. Schrier and Dr. L. P. Lipsitt respectively. 